\title{
Widths of Lindblad Resonances in Galaxies: Origin of the Hole in the Gas Disk of the Milky Way
}

\author{
Leonid Marochnik \\ Computer Sciences Corporation, Lanham, Maryland, 20706 \\ Roald Sagdeev and Daniel Usikov \\ Department of Physics, University of Maryland, College Park, \\ Maryland, 20742-3280
}

\begin{abstract}
It is shown that slopes of $p(R)=\Omega(R) \pm \kappa(R) / m$ curves at Lindblad resonances determine the widths of resonance regions. The ability of galactic disks to respond to torques exerted at ILRs by perturbers (bar, density wave, galaxy-satellite, etc.) is determined by the widths of inner Lindblad resonances (ILRs). Widths of ILRs vary along the Hubble sequence of normal and barred galaxies. Galaxies having the bulge to disk ratio of masses and radii similar to the Milky Way could have wide ILRs if they are formed at the region of $2-4 \mathrm{kpc}$ from their centers. A wide range of possible perturbers with pattern speeds $4 \leq \Omega_{p}$ $\leq 26 \mathrm{~km} \mathrm{~s}^{-1} \mathrm{kpc}^{-1}$ could excite an ILR at this region of the Milky Way. Probably, the ILR of Milky Way's grand design is located in the same region. The hole in the galactic $\mathrm{H}_{2}$ disk is also located in this region. The mechanism responsible for the origin of this hole could be similar to that opening gaps in planetary rings.
\end{abstract}

\section{Width of an Inner Lindblad Resonance}

The position of a Lindblad resonance (LR) is determined by the condition $\Omega_{p}=$ $p(R)$ for $p(R)=\Omega(R) \pm \kappa(R) / m$, where $\Omega_{p}$ is the phase angular velocity of an external perturber, and $\Omega(R)$ and $\kappa(R)$ are the angular and epicyclic frequencies of a particle at the distance $R$ from the center of gravitational field. Let us assume that a particle "feels" an LR in the band width of a perturber's frequency $\Delta \Omega_{p}$. If so, one can write $\Delta \Omega_{p}=\left.\Delta R(d p / d R)\right|_{L R}$ and the LR width $\Delta R / R$ is:

$$
\left|\frac{\Delta R}{R_{L R}}\right|=\left|\frac{\Delta \Omega_{p} / \Omega_{p}}{d l n p / d l n R}\right|_{R=R_{L R}} \equiv\left|\frac{\Delta \Omega_{p} / \Omega_{p}}{q\left(R_{L R}\right)}\right|
$$

Equation (1) shows that the width of LRs increases with decreasing slope $q(R)=d(\ln p) / d(\ln R)$ of the curve $p(R)$ at the LR position. To define the term "a particle feels" (see above) we calculate the mean displacement of a particle from its initial undisturbed circular orbit at the position of the ILR. Using the standard theory of perturbations one can find (Marochnik \& Usikov 1996): 


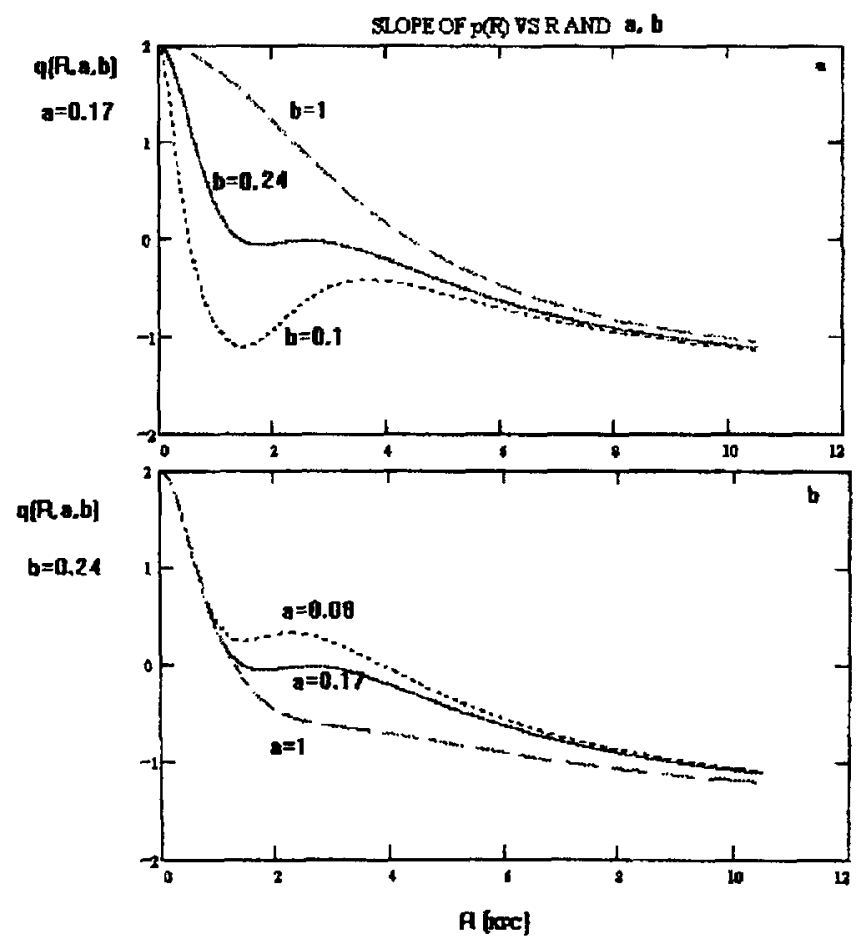

Figure 1. Curves $q(R)=d(\ln p) / d(\ln R)$ for a simple galactic model consisting of a Toomre disk+spheroid with ratios of their masses and radii $a=M_{s} / M_{d}$ and $b=R_{s} / R_{d}$. Both (a) and (b) panels demonstrate a plateau in the $q$-curve near its zero at the $2-4 \mathrm{kpc}$ region for the Milky Way's $a$ and $b$ parameters.

$\rho=\frac{\langle r\rangle}{\left\langle r_{0}\right\rangle}=\left|\frac{\sin \left(\frac{2 \pi}{\kappa}\left(p(R)-\Omega_{p}\right)\right)}{\frac{2 \pi}{\kappa}\left(p(R)-\Omega_{p}\right)}\right|=\left|\frac{\sin \left(\frac{2 \pi}{\kappa} \Delta R \frac{d p}{d R}\right)}{\frac{2 \pi}{\kappa} \Delta R \frac{d p}{d R}}\right|_{R=R_{L R}}=\left|\frac{\sin \left(\frac{2 \pi}{\kappa} \Delta \Omega_{p}\right)}{\frac{2 \pi}{\kappa} \Delta \Omega_{p}}\right|$

where $\left\langle r_{0}\right\rangle$ is the mean displacement of the particle from its initial circular orbit (averaged on its epicyclic time period $2 \pi / \kappa$ ) that is located at the ILR position $\left(\Delta R=\Delta \Omega_{p}=0\right)$ and $\langle r\rangle$ is the mean displacement of the particle from its initial circular orbit located at the distance $\Delta R$ from the ILR. One can find from Equation (2) that $\Delta \Omega_{p} / \kappa \approx 6^{1 / 2}(1-\rho)^{1 / 2} / 2 \pi$ for $1-\rho<<1$. Thus, the displacement $(1-\rho)^{1 / 2}$ of a particle from its resonance orbit $(\rho=1)$ governs the band width of the perturber's frequency $\Delta \Omega_{p}$ that this particle "feels" near the ILR.

The ability of galactic disks to respond to torques exerted at ILRs by external perturbers (bars, galaxy-satellites, etc.) is governed by the magnitude of the 


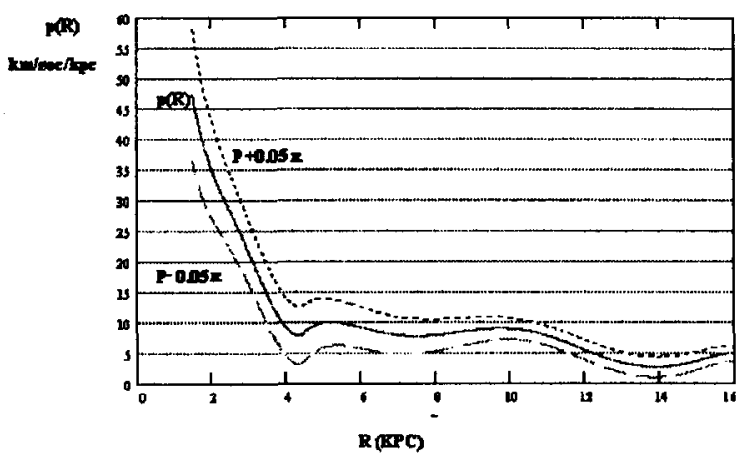

Figure 2. Curve $p(R)=\Omega(R)-\kappa(R) / 2$ for the Milky Way calculated from the Clemens' (1985) data. Upper and lower curves show the band width $\Delta \Omega_{p}(R)= \pm 0.05 \kappa(R)$. Particles (stars, gas clouds) "feel" an ILR inside this band with $98 \%$ accuracy.

$q(R)$ function at the ILR position, i.e. it is governed by the width of the ILR. It is because the torque of an external perturber is proportional to $q^{-1}$ (Goldreich \& Tremaine 1979), i.e. it is proportional to $\Delta R$ (see Equation (1)). To consider how widths of ILRs vary along the Hubble sequence of galaxies we study variations of their $q(R)$ curves, i.e. slopes of their $p(R)$ curves. We consider a simple model of a galaxy consisting of a spheroid and a Toomre disk with the ratios of their masses and radii $a=M_{s} / M_{d}$ and $b=R_{s} / R_{d}$, respectively. Varying these $a$ and $b$ parameters, we can study the behavior of $p(R)$ slopes of models that correspond qualitatively to the Hubble sequence. Figures $1 \mathrm{a}$ and $\mathrm{b}$ show that models containing more compact and more massive spheroidal components have zones of small $|q|$ at distances closer to their centers and vice versa. Thus, the efficacy of ILRs depends on their positions in galaxies of different types. We expect to find wide ILRs close to the centers of Sa galaxies, whereas in Sc galaxies the wide ILRs should be situated farther away from their centers.

Figure 1 demonstrates also that there is a wide zone of small $q$ (plateau in the $q$-curve near its zero) at the $2-4 \mathrm{kpc}$ region for a model with parameters $a=$ 0.17 and $b=0.24$ that are close to the Milky Way's $a$ and $b$ parameters. These estimates correspond to $M_{s}=10^{10} M_{\odot}, R_{s}=1 \mathrm{kpc}$ (mass and radius of the central bulge) and $M_{d}=6 \times 10^{10} M_{\odot}, R_{d}=3.5 \mathrm{kpc}$ which could be accepted for the Milky Way (Bahcall 1986).

\section{Inner Lindblad Resonances in the Milky Way}

To consider widths of possible ILRs in the Milky Way (MW) we employ the polynomial approximation of its rotation curve by Clemens (1985). Using the Clemens' approximation for the Sun's position, $R_{o}=10 \mathrm{kpc}$, one can find $p(R)$ which is shown in Figure 2. 


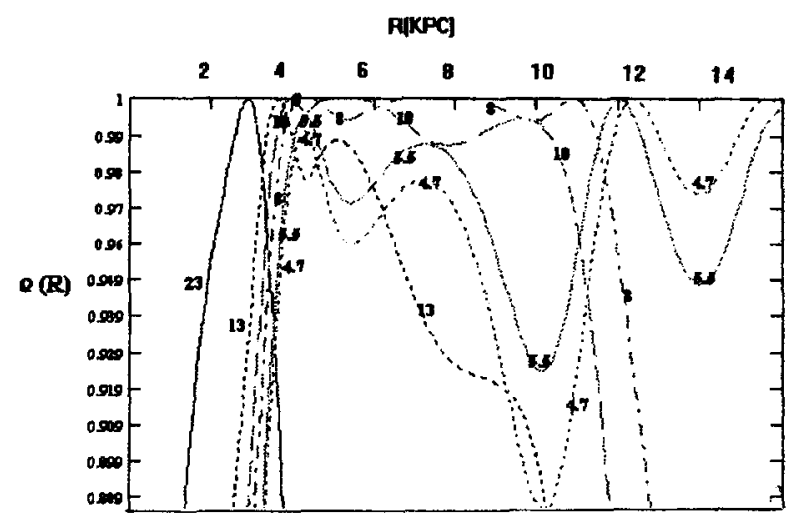

Figure 3. Function $\rho\left(R, \Omega_{p}\right)$ from Equation (2) for the Milky Way (see text). Numbers inside the panel are $\Omega_{p}$ values. This figure shows widths of ILRs for $\Omega_{p}$ values of interest.

The Milky Way's $p(R)$-curve shows a number of special features. Let us note, e.g., that the ring of molecular hydrogen is situated between two minima of $p(R)$ at 4 and $8 \mathrm{kpc}$. The MW could have its IILR and OILR at these regions if $\Omega_{p}$ of its grand design (or possible bar) is close to $8 \mathrm{~km} \mathrm{~s}^{-1} \mathrm{kpc}^{-1}$. However, it is not to be supposed that an exact value of the $\Omega_{p}$ at this region is crucial because the MW's $p(R)$ curve is practically flat at the region between 4 and 10 kpc (see Figure 2) as it was first noted by Lindblad (1958). This means that nonresonant particles (stars and gas clouds) could "feel" an ILR at this zone at the wide band width $\Delta \Omega_{p}$. The upper and lower curves in Figure 2 are shown for $\Omega_{p}(R)= \pm 0.05 \kappa(R)$. This band width corresponds to $1-\rho=0.02$ in Equation (2) as the displacement of a particle from its orbit located at an exact resonance is only $2 \%$. One can see from Figure 2, for example, that nonresonant particles "feel well" (with $98 \%$ accuracy) a resonance in the band width of $\Omega_{p}$ from 3 to $13 \mathrm{~km} \mathrm{~s}^{-1} \mathrm{kpc}^{-1}$ near $\mathrm{R}=4 \mathrm{kpc}$.

Figure 3 shows the widths of ILRs in the MW corresponding to $\Omega_{p}$ values of interest. Figure 3 shows that particles are able to "feel" in a resonance manner a wide spectrum of possible external perturbers $\left(4 \leq \Omega_{p} \leq 26 \mathrm{~km} \mathrm{~s}^{-1} \mathrm{kpc}^{-1}\right)$ at the $2-4 \mathrm{kpc}$ region. Because of this wide band width, ILRs could be formed at this region even for $\Omega_{p}$ values which do not intersect the $p(R)$ curve (e.g., for $\Omega_{p}$ $\left.\approx 4-7 \mathrm{~km} \mathrm{~s}^{-1} \mathrm{kpc}^{-1}\right)$. Note that $\Omega_{p}=5.5 \mathrm{~km} \mathrm{~s}^{-1} \mathrm{kpc}^{-1}$ is the angular velocity of the Blitz \& Spergel (1991) bar; $\Omega_{p}=4.7 \mathrm{~km} \mathrm{~s}^{-1} \mathrm{kpc}^{-1}$ is the frequency which could be excited by the LMC tidal force in the Milky Way (Marochnik \& Suchkov 1996). Binney et al.'s (1991) and Blitz et al.'s (1993) bar of $\Omega_{p}=63$ $\mathrm{km} \mathrm{s}^{-1} \mathrm{kpc}^{-1}$ has its corotation at $2.4 \mathrm{kpc}$ that, probably, is close to the ILR position of the MW grand design (Marochnik \& Suchkov 1996). For this special situation $\Omega_{p}$ values of the bar and grand design could not coincide (Tagger et al. 1987). 
Thus, the $2-4 \mathrm{kpc}$ region in the MW is a place where a wide ILR could be located for a very wide spectrum of possible $\Omega_{p}$. This is also the place of the hole location in the galactic $\mathrm{H}_{2}$ disk. The mechanism responsible for the opening of this hole could be similar to that responsible for the open gaps in the planetary rings. The density wave excited in the system of molecular clouds by an external perturber (e.g., gravitational potential of the spiral grand design, bar, etc.) has to damp due to cloud collisions. The negative angular momentum of this wave would transfer to the system of molecular clouds. The clouds just outside ILR would spiral inward and open a hole at the region of $2-4 \mathrm{kpc}$ from the galactic center (Marochnik \& Usikov 1996).

\section{References}

Bahcall, J. 1986, ARA\&A, 24, 577

Binney, J. J., Gerhard, O. E., Stark, A. A., Bally, J., \& Uchida, K. I. 1991, MNRAS, 252, 210

Blitz, L. \& Spergel, D. 1991, ApJ, 370, 205

Blitz, L., Binney, J., Lo, K. Y., Bally, J., \& Ho, P. T. P. 1993, Nature, 361, 417

Clemens, D. 1985, ApJ, 295, 422

Goldreich, P. \& Tremaine, S. 1979, ApJ, 233, 857

Lindblad, B. 1958, Stoc. Obs. Ann. 20, No.6

Marochnik, L. \& Suchkov, A. 1996, The Milky Way Galaxy, New York: Gordon \& Breach, in press

Marochnik, L. \& Usikov, D. 1996, in preparation

Tagger, M., Sygnet, J. F., Athanassoula, E., \& Pellat, R. 1987, ApJ, 318, L43 